\title{
Jouer avec l'anglais juridique
}

\section{Barbara S. Villez}

\section{(2) OpenEdition \\ Journals}

Édition électronique

URL : http://journals.openedition.org/asp/4103

DOI : 10.4000/asp.4103

ISSN : 2108-6354

\section{Éditeur}

Groupe d'étude et de recherche en anglais de spécialité

\section{Édition imprimée}

Date de publication : 1 décembre 1994

Pagination : 237-242

ISSN : 1246-8185

\section{Référence électronique}

Barbara S. Villez, « Jouer avec l'anglais juridique », ASp [En ligne], 5-6 | 1994, mis en ligne le 19 décembre 2013, consulté le 30 avril 2019. URL : http://journals.openedition.org/asp/4103 ; DOI : $10.4000 / a s p .4103$

Ce document a été généré automatiquement le 30 avril 2019.

Tous droits réservés 


\title{
Jouer avec l'anglais juridique
}

\author{
Barbara S. Villez
}

1 Pour se servir d'une langue spécialisée, il faut acquérir un vocabulaire spécialisé et avoir maîtrisé la grammaire de cette langue. La pratique d'une langue de spécialité permet de comprendre tous ceux qui utilisent cette langue professionnellement, ceux dont c'est la langue maternelle et ceux d'autres nationalités. Parfois, on doit utiliser la langue de spécialité pour présenter une communication à un colloque par exemple, ce qui est impressionnant même dans sa propre langue. Bien que tout le monde ne soit pas amené à se faire publier dans des revues de spécialité à l'étranger, tous ceux qui pratiquent une langue de spécialité l'utilisent pour lire des documents authentiques et entretenir une correspondance.

2 Il est évident que toutes ces activités présupposent une bonne connaissance de la grammaire de la langue et de la culture du pays, et incluent l'acquisition du vocabulaire de la spécialité. Dans l'idéal, la première de ces pré-exigences aura été acquise dans l'enseignement secondaire, ce qui permet aux étudiants du DEUG, par exemple, de se concentrer sur les deux autres. Malheureusement, le niveau de langue de ces étudiants est souvent tel que la pratique de la langue de spécialité doit s'accompagner d'une sérieuse révision de la grammaire fondamentale. On peut ainsi faire travailler des étudiants sur les documents de leur spécialité : futurs médecins sur des articles de revues de médecine, informaticiens sur des programmes écrits dans la langue cible, etc.

De telles activités mettent en évidence divers aspects de la culture. Les étudiants se concentrent tantôt sur l'apport culturel d'un article, tantôt sur l'apport lexical, tantôt encore sur ses structures syntaxiques. Les futurs spécialistes sont capables de noter des nuances culturelles dans un article dès lors qu'ils ont déjà étudié les fondements techniques de leur spécialité dans leur propre langue. Pour un mathématicien, les notions mathématiques ne changent pas d'une langue à une autre; simplement la langue, le vocabulaire et éventuellement certaines expressions seront différents. Tous les médecins du monde traitent le même corps humain. Certains soins peuvent varier selon les pays, mais les notions médicales restent équivalentes - même si les pratiques cliniques (culturelles ?) diffèrent parfois. 
Dans d'autres professions, les différences d'ordre culturel sont d'emblée évidentes. Le juriste français par exemple découvre rapidement que toute sa connaissance du droit français ne facilite pas sa lecture d'articles sur le common law britannique. Si on traduit " avocat » par "lawyer», on ne tient pas compte du fait qu'un avocat anglais est, malgré la dernière réforme de 1990, soit un « solicitor » soit un «barrister ». Un « solicitor » et un " barrister " ne remplissent pas les mêmes fonctions, ne suivent pas la même formation et n'ont pas le même parcours de carrière. Un «magistrat » français préside souvent une Cour après avoir accompli ses études initiales à l'École de la magistrature. Par conséquent, lorsque l'étudiant français apprend qu'il est injurieux de traiter un «judge » anglais de " magistrate», sa perplexité est grande. En effet, non seulement un « magistrate " anglais n'est pas un "judge », mais seul ce dernier a suivi une très longue formation juridique.

\section{Anglais juridique}

5 L'enseignement de l'anglais juridique présente donc des problèmes majeurs dans les universités françaises. Le premier relève de la nécessité de fonder l'enseignement de la langue de spécialité sur une culture juridique qui permettrait de comprendre un système différent, et ensuite en emprunter les termes techniques à bon escient. Un deuxième problème de taille surgit lorsqu'on considère que les étudiants doivent à la fois aborder un nouveau système juridique et faire l'apprentissage d'un nouveau vocabulaire spécialisé dans une langue qu'ils possèdent assez mal. En effet, le niveau d'anglais de la majorité d'étudiants en DEUG de droit est, après sept ans d'apprentissage d'anglais, encore très médiocre, voire insuffisant. Tout ceci encombre le faible nombre d'heures destinées à l'étude de l'anglais juridique d'un contenu extrêmement lourd. D'autres problèmes existent, mais nous nous limiterons ici à celui que présente la difficulté qu'éprouvent les enseignants face à ce triple contenu : langue, vocabulaire juridique et culture juridique. Nous proposons comme solution - ne serait-ce que partielle - la technique qui consiste à jouer avec l'anglais juridique.

6 L'explication de la différence entre «judge » et « magistrate » impressionne peu l'étudiant gaffeur. Combien d'explications sont bien comprises et ensuite oubliées? Lorsqu'ils jouent, les étudiants ont un rôle actif et ils sont plus facilement frappés par les conséquences de leurs actes. De plus, il est agréable de jouer si l'activité est spontanée. L'acte de jouer est également un moyen d'enculturation. Les attitudes, les habitudes et l'humour d'un peuple sont transmis par ses jeux et ses façons de jouer. Lorsque les étudiants jouent avec la langue, une complicité se crée entre eux. Ces activités favorisent des échanges langagiers et la production spontanée de la langue.

7 Il est fréquent de jouer lorsqu'on apprend une langue. Le «bon apprenant " a une disposition ludique, ce que les Anglais appellent « a playful attitude » envers la langue qu'il apprend. Lorsqu'on joue, on développe cette disposition ludique. Les psychologues définissent la disposition ludique comme un comportement caractérisé par la spontanéité physique, sociale et cognitive, un sens de l'humour et de la gaîté (Lieberman 1977: 5) ${ }^{1}$. Cette disposition s'apparente à une sorte d'espièglerie ou de légèreté de cœur. Lorsqu'on lance un défi à ses étudiants, il faut le faire dans un tel esprit. Le jeu s'accompagne toujours de rigueur (les règles). Il peut donc $\mathrm{y}$ avoir une place pour les règles de grammaire dans une ambiance de jeu. 
Dans les cours d'anglais juridique, les apprenants doivent en même temps manipuler le nouveau vocabulaire spécialisé, maîtriser les notions du droit anglais, et se concentrer afin de construire des phrases correctes ; ceci les perturbe au point qu'ils finissent parfois par utiliser des mots anglais pour décrire le système français. À un examen, ceci est certes dommageable, mais dans une situation professionnelle, cela devient beaucoup plus grave. Cependant, dans le cadre d'un cours, un enseignant peut imaginer des stratégies incitant ses étudiants à se rendre compte de cette confusion et à se corriger.

\section{Jouer avec la culture juridique de base}

9 S'indigner d'une façon ludique à la place du « judge » qui s'est fait traiter de "magistrate » aura plus d'effet sur les étudiants que ne pourrait en avoir une explication de la différence entre ces deux fonctions. Quand on demande aux étudiants de trouver par euxmêmes la raison de l'indignation du «judge », le défi stimule leur curiosité. En outre, leur surprise devant la réaction du «judge » restera certainement plus longtemps en mémoire qu'une explication.

Un «magistrate» n'est pas un homme de loi. Pour devenir «judge» il faut avoir été « barrister » : les années de plaidoirie au barreau sont considérées comme la seule formation possible. En dépit de la réforme dénommée Courts and Legal Services Act de 1990, qui autorise en principe des « solicitors » à devenir des « judges » inférieurs, actuellement seuls les «barristers» peuvent devenir des «judges», inférieurs ou supérieurs. Pour comprendre la logique de cette tradition, le juriste français doit savoir qu'un "barrister " prépare sa plaidoirie essentiellement à partir de la jurisprudence. Il doit convaincre le « judge » de la Cour, (au procès, il n'y en a qu'un, à la différence de la France), que les faits du cas qu'il plaide sont les mêmes que ceux d'un «precedent ». Si le «judge » est d'accord, il est obligé d'appliquer la même décision, une décision qui serait favorable au client du " barrister ». Ainsi les « barristers » et les «judges » doivent-ils avoir une bonne connaissance de la jurisprudence anglaise. Il est impératif qu'un juriste français sache comment les « judges» prennent leurs décisions et comment de telles décisions jurisprudentielles deviennent lois. Rien de comparable n'existe en droit français.

11 Les étudiants ont tendance à devenir passifs lors des explications qu'on peut donner sur le système anglais. Plus on y passe de temps, plus on risque la répétition, ce qui est générateur d'ennui. Cependant, quand il s'agit de fournir aux étudiants les éléments utiles à la résolution du « défi » qui leur est lancé, on peut passer le temps nécessaire sur les différents aspects du système juridique anglais tout en les maintenant actifs. L'enseignant, ainsi davantage en retrait, peut vérifier dans quelle mesure les étudiants ont réellement assimilé ces différences culturelles juridiques. Par exemple, que feraientils du fait que les «judges » ne s'occupent que de $10 \%$ de toutes les affaires criminelles et que les « magistrates » s'occupent du reste?

12 Le fait de jouer avec ces notions de culture juridique permet plus de variété. En modifiant les défis, l'enseignant obtient que les étudiants manipulent et remanipulent le contenu. Par exemple, il peut écrire au tableau une phrase à propos d'un "magistrate » et ensuite demander aux étudiants d'effectuer tous les changements nécessaires si on remplaçait « magistrate» par "judge». D’autres changements peuvent être effectués ensuite et l'enseignant demandera aux étudiants d'essayer de remettre «magistrate » dans la phrase. 
Un exercice de ce type appelle une réflexion sur la langue ainsi que sur les notions culturelles.

13 L'élément essentiel de ces activités est le défi. Les étudiants s'engageront dans les activités qui leur sont proposées s'ils reconnaissent la présence de défis. Ils seront actifs et développeront des stratégies qui pourraient leur servir lorsqu'ils se trouveront dans des situations professionnelles. C'est leur poser un défi que de leur demander de justifier pour quelles raisons un contrat est considéré comme nul pour défaut de "consideration ». Ils découvriront rapidement qu'ils ne pourront pas se débarrasser du problème en traduisant ce terme par «contrepartie ». En effet, tous les contrats incluent ce terme, mais les cours anglaises soumettent les contrats à des règles très strictes pour déterminer la présence ou l'absence de "consideration ». L'étude du vocabulaire juridique ne peut pas se dissocier de l'étude de la culture juridique anglaise de base. Si l'on ne maittrise pas les deux, on est incapable de comprendre les documents juridiques anglais, et plus particulièrement les longs arrêts qui constituent une partie importante de la loi anglaise, une lecture courante pour les juristes anglais. En jouant avec les notions sous-jacentes à l'anglais juridique, l'étudiant français en acquiert une expérience active.

14 Si les activités proposées ici comme exemples n'évoquent pas le «jeu » à proprement parler, c'est peut-être parce que, dans les cours de langues, on associe habituellement ce terme avec "simulation" ou bien «jeu court et structuré », à but très déterminé, activités visant à renforcer le contenu d'une leçon. Ces types de jeu ne satisfont pas tous les publics. Parfois, les apprenants se sentent mal à l'aise dans les jeux de rôle. De plus, certains groupes sont trop importants pour que de tels jeux soient efficaces. Les activités ludiques spontanées («play» en anglais, et non "games») correspondent mieux aux intérêts et à la personnalité des apprenants dans un groupe, parce qu'eux-mêmes participent à la création de ces activités. Il n'y a pas que les enfants qui sont autorisés à jouer. Nous proposons plus loin d'autres exemples d'activités qui peuvent servir comme point de départ.

\section{Jouer : une technique qui permet d'alléger la lourdeur des tâches}

Les adultes en formation permanente présentent une grande diversité de niveaux en anglais et certains de ces adultes n'ont pas étudié l'anglais depuis de longues années. Les étudiants en DEUG de droit dans les universités françaises couvrent également une vaste gamme de niveaux en anglais, et pour une grande majorité sont incapables d'appliquer les règles grammaticales lorsqu'ils se concentrent sur le sens de leurs énoncés écrits ou oraux. Lorsqu'on joue, on s'exprime. L'expérience active et la vive cadence des activités ludiques favorisent le développement des automatismes, la maîtrise des structures grammaticales de la langue.

16 Lorsqu'on joue avec l'anglais juridique l'impression qui en résulte est de diminuer l'énormité du programme. Les activités peuvent focaliser les efforts tantôt sur la langue, tantôt sur le contenu juridique tout en exigeant une production grammaticalement correcte. Ces activités font travailler simultanément les trois éléments de l'anglais juridique, mais en déplaçant l'effort à chaque fois, ce qui donne l'impression d'un travail plus abordable. 
17 L'erreur fait partie du jeu. Si l'on se trompe, on comprend qu'il faut effectuer un changement dans ses actions, dans ses stratégies. En classe, on craint l'erreur, mais dans le sport, dans le jeu, dans toute activité ludique, on l'accepte plus facilement et on s'en sert pour améliorer sa performance. Dans les cours de langue, l'erreur est fréquente, alors mieux vaut encourager une attitude plus neutre envers elle.

\section{La technique de jeu pour aider les enseignants}

18 Le jeu seul ne permet pas de résoudre tous les problèmes liés à la diversité des niveaux de langue des étudiants en DEUG. Même si les étudiants sont placés en groupes de niveaux, il s'agit de déterminer qui doit enseigner à ces différents groupes et comment s'y prendre? Y a-t-il un ordre de priorités ou faut-il faire travailler le triple contenu simultanément ? Cette deuxième solution est plus pragmatique et permet aux enseignants d'anglais juridique de penser que leurs cours correspondraient mieux aux intérêts et goûts des futurs juristes. Cependant, qui enseignera mieux un tel contenu, un juriste ou un linguiste?

19 Le linguiste, ne pouvant pas se servir de ses compétences propres, se sent obligé d'acquérir rapidement des notions juridiques pour rester un peu en avance sur ses étudiants. Il arrive que dans le souci de maintenir la présentation de la langue dans un contexte juridique, le linguiste privilégie le droit au détriment de la langue dans son enseignement. On retrouve, dans ces cours, des discussions, à partir d'un article de journal par exemple, limitées à l'expression des opinions de quelques-uns des membres du groupe. De peur de décourager les volontaires, les erreurs ne sont pas signalées ou très peu. Une autre possibilité est que la discussion frustre les étudiants plus avancés qui voudraient poursuivre le sujet de façon plus technique que la culture juridique de leur enseignant ne le permet.

20 L'avocat, en revanche, est plus à l'aise dans les discussions sur le droit, mais il est mal placé pour résoudre les problèmes linguistiques de ses étudiants ou assurer leur production spontanée de la langue. Les juristes finissent souvent par donner des cours magistraux où le seul qui parle est le seul qui n'a point besoin de pratique. Il ne faut pas oublier que les cours d'anglais juridique sont des cours de langue avant tout. Qu'ils permettent aux futurs juristes français d'assister à des conférences en anglais est important certes, mais ils doivent les entraîner à pouvoir participer activement à des échanges professionnels.

21 Au Département de langues juridiques et économiques de l'Université de Paris 2, des juristes de langue maternelle anglaise sont chargés des cours de niveau trois, pour les étudiants plus avancés. Les cours de niveaux faible et moyen, plus centrés sur la grammaire et la correction, sont dispensés par des linguistes. Les étudiants de niveau trois travaillent sur des documents authentiques avec le juriste et les discussions sont plus techniques. L'équipe enseignante de Paris 2 a constaté que les documents authentiques offraient peu de possibilités pédagogiques pour les groupes des deux autres niveaux. Les juristes et les linguistes de l'équipe ont collaboré alors à la création d'un programme minimum de culture juridique anglaise pour ces étudiants.

Il fallut d'abord déterminer des connaissances requises du système juridique anglais. Ceci fait, des juristes ont écrit une série d'articles courts sur des sujets tels que les sources du droit anglais, les professions juridiques, la hiérarchie des juridictions et la procédure. 
D'autres articles ont été écrits plus tard pour permettre un travail sur le système fédéral des États-Unis. Des juristes ont repris le contenu de ces articles et en ont tiré une deuxième série de textes simplifiés. Il était parfois nécessaire de réorganiser les informations afin d'utiliser certaines structures grammaticales ou de présenter des motsclés suivis d'explications ou de définitions. Ces deux séries de textes sont suivies d'autres articles pour des lectures approfondies et d'exercices qui visent l'exploitation des structures employées dans les textes simplifiés.

Les exercices qui accompagnent ce matériel sont fondés sur la technique de jeu car le troisième avantage qu'une approche ludique apporte à l'enseignement de l'anglais spécialisé est d'aider les enseignants à ne pas trop interférer avec des problèmes qui surgissent en dehors de leur propre domaine de spécialisation. Grâce à une disposition ludique, le juriste peut refuser de répondre aux questions avant qu'elles ne soient reformulées correctement, plutôt que de se lancer dans des explications maladroites de la grammaire anglaise. Par le jeu, le linguiste se fera guider dans le découvert du droit anglais par ses étudiants-juristes français. Il peut le faire en imposant certaines limites à leur activité, des limites comme il en existe dans toute activité ludique; cela peut être l'exigence de l'expression grammaticalement correcte ou l'utilisation obligatoire de certains mots techniques dans les phrases des étudiants. Le jeu libère les enseignants de l'obligation de détenir toutes les réponses.

Les trois activités qui suivent sont proposées dans le but d'indiquer comment on pourrait jouer avec l'anglais juridique. Ce ne sont que des exemples d'exercices à but ouvert, où plusieurs réponses sont possibles à chaque fois. Il est espéré que ces activités déclencheront des idées de jeux alternatifs aux jeux habituellement utilisés dans les cours de langue.

I. Make as many sentences as you can using only the words listed here. You may use any form of the verbs and plurals of nouns as well as the possessive forms of nouns; for example, plead, pleaded, pleads; solicitor, solicitors, solicitor's, solicitors'.

when/ plaintiff/ barrister/ if/ never/ solicitor/ court/ advise/ can/ who/ by/ must/ a/ not/ want/ the/ case/ to/ of/ start/ settle/ against/ plead/ person/ he/ another/ consult/ engage/ is/ his/ out/ and/ sometimes/ in/ be/ client/ him/ directly/ (Cochrane et al. $1979: 63$ )

II. How many different affinity groups of words can you make form the following list?

murder/ civil/ case/ trial/ rape/ criminal/ administer/ probate/ appeal/ try/ recovery/ estate/ defendant/ will/ justice of the peace/ indictable/ damages/ rob/ sue/ punish/ summary/ contest/ defend/ handle/ original jurisdiction/ guardianship/drunkenness/ enquiry/ belong/ offence/ breach of contract/ plaintif f/ compensate/ (Cochrane et al. $1979: 85$ )

III. Use each of the following words in a separate sentence: judicial - judiciary - jurisdiction - jurist - judge - justice - juror (Fourquet et al. 1986 : 79)

Write sentences in which you use three of the above at the same time. Try to use all of the words in one sentence. Write a short paragraph using all these words.

Il y a en effet beaucoup de possibilités. Ce qui compte avant tout, c'est la présence d'un défi dans les activités qu'on propose. Lorsque les défis deviennent trop faciles, attestant le progrès des apprenants, il faut les modifier. Les étudiants sont amenés à utiliser la langue pour accomplir des tâches précises. À force de manipuler et remanipuler les mêmes concepts, les étudiants les apprennent sans vraiment s'en rendre compte. Ainsi, la technique de jeu possède un aspect subversif. 
Il y a une raison supplémentaire d'adopter une approche quelque peu subversive. Les apprenants peuvent avoir des idées assez traditionnelles en ce qui concerne le moment et l'endroit pour jouer. Il est rarement avisé de présenter un exercice comme un jeu et surtout pas avec des étudiants de DEUG. Les étudiants n'ont pas besoin de considérer une activité comme un jeu pour relever un défi. En fonction de leurs progrès, l'enseignant modifie les défis. Il vérifie s'ils ont réellement compris un concept ou maîtrisé une structure en essayant de les piéger gentiment. Il est utile que les enseignants développent une approche plus ou moins espiègle pour encourager une disposition ludique chez leurs étudiants dans leur approche de l'apprentissage de la langue.

Lorsqu'une langue est enseignée comme un ensemble de connaissances, la production spontanée de la langue devient pratiquement impossible. Il est difficile d'éviter cette situation dans l'enseignement de l'anglais juridique. Pour permettre aux avocats d'utiliser une langue étrangère de façon efficace dans un contexte professionnel, il faut qu'ils maîtrisent l'utilisation spontanée de la langue aussi bien que la compréhension de celleci. La technique de jeu facilite la spontanéité et aide les apprenants à développer les moyens d'étendre leur apprentissage au-delà des activités de la classe.

\section{BIBLIOGRAPHIE}

Cochrane, M. et al. 1979. Legal English I. Paris : Éditions Cujas.

Fourquet, M. et al. 1986. Legal English II. Paris : Éditions Cujas.

Lieberman, J. Nina. 1977. Playfulness: Its relationship to imagination and creativity. New York:

Academic Press.

\section{NOTES}

1. «Playfulness... [is behavior which can be characterised by] physical, social and cognitive spontaneity, sense of humour, and manifest joy ».

\section{RÉSUMÉS}

Théorie et pratique du droit sont très différentes en France et en Grande-Bretagne. La langue de spécialité à acquérir suppose une bonne compréhension de ces concepts culturellement différents. Des techniques pédagogiques ludiques aident fortement à cette acquisition. 
The theory and practice of law are very different in France and in Britain. It is necessary to have a good understanding of the underlying culturally different concepts when learning English for law. It can be exceedingly helpful to use pedagogical "play" techniques.

INDEX

Mots-clés : concept juridique, langue du droit, pédagogie

Keywords : concept in law, language of law, pedagogy

\section{AUTEUR}

\section{BARBARA S. VILLEZ}

Barbara S. Villez enseigne à l'Université Paris 2. villez.barbara@orange.fr 